\title{
Probe electrode study of cathodically polarized PtIr-YSZ interfaces
}

\author{
Hansen, Karin Vels; Kreka, Kosova; Jacobsen, Torben
}

Published in:

Journal of Solid State Electrochemistry

Link to article, DOI:

10.1007/s10008-018-04179-0

Publication date:

2019

Document Version

Peer reviewed version

Link back to DTU Orbit

Citation (APA):

Hansen, K. V., Kreka, K., \& Jacobsen, T. (2019). Probe electrode study of cathodically polarized PtIr-YSZ

interfaces. Journal of Solid State Electrochemistry, 23(3), 811-822. https://doi.org/10.1007/s10008-018-04179-0

\section{General rights}

Copyright and moral rights for the publications made accessible in the public portal are retained by the authors and/or other copyright owners and it is a condition of accessing publications that users recognise and abide by the legal requirements associated with these rights.

- Users may download and print one copy of any publication from the public portal for the purpose of private study or research.

- You may not further distribute the material or use it for any profit-making activity or commercial gain

- You may freely distribute the URL identifying the publication in the public portal

If you believe that this document breaches copyright please contact us providing details, and we will remove access to the work immediately and investigate your claim 


\author{
Probe electrode study of cathodically polarized PtIr-YSZ interfaces \\ Karin Vels Hansen ${ }^{\mathrm{a}}$, Kosova Kreka ${ }^{\mathrm{a}}$ and Torben Jacobsen ${ }^{\mathrm{b}}$ \\ ${ }^{a}$ Department of Energy Conversion and Storage, Technical University of Denmark, \\ DK-4000 Roskilde, Denmark \\ ${ }^{b}$ Department of Chemistry, Technical University of Denmark, DK-2800, Kgs. Lyngby, Denmark
}

Corresponding author: Karin Vels Hansen, karv@dtu.dk, Tel. +45 46775796, ORCID: 0000-0001-7169-2102

\begin{abstract}
Local cathodic polarizations of yttria-stabilized zirconia were carried out with a PtIr probe as the working electrode in a controlled atmosphere high temperature scanning probe microscope to investigate the reduction of zirconia. Impedance spectroscopy was performed at $650^{\circ} \mathrm{C}$ during increasing and decreasing polarization, in a range between $0.5 \mathrm{~V}$ and $-2 \mathrm{~V}$ in $9 \% \mathrm{H}_{2}$ in $\mathrm{N}_{2}$ saturated with water vapor at room temperature $\left(25^{\circ} \mathrm{C}\right)$. With increased polarization, the impedance spectra changed from a simple suppressed arc at low polarizations into two capacitive arcs separated by an inductive loop and followed by an inductive loop at low frequencies. Areas with high conductance as well as significantly decreased high frequency resistances resulted from the polarizations and indicate the introduction of electronic conductivity in YSZ. Near the probe |YSZ contacts, areas with very low conductance and accumulation of Sicontaining particles were observed, pointing to additional migration of silica impurities towards the probe.
\end{abstract}

Keywords: PtIr-YSZ, Strong cathodic polarization, inductive impedance, CAHT-SPM

\title{
Introduction
}

Solid oxide electrolysis cells (SOEC) are essential for future energy storage systems, but presently they experience different types of degradation when running at high current densities. Whereas solid oxide fuel cells operate safely within the stability region of the yttria-stabilized zirconia electrolyte (YSZ), the electrode potential of the Ni-YSZ cathode in SOEC can - at high current densities - become very low and induce electronic conductivity in YSZ accompanied by a partial reduction of zirconia. These phenomena are closely related to the so called "blackening" of zirconia [1].

Strong negative polarization of $\mathrm{Ni}$-YSZ electrodes has been shown to result in microstructural and compositional changes of the materials $[2,3]$ causing severe degradation for SOECs, but very short treatments have been shown to improve the microstructure and performance of SOFC anodes $[4,5]$. A partial reduction of zirconia and YSZ occurs already to a mild extent above $200^{\circ} \mathrm{C}$ in hydrogen [6], in atmospheres where the oxygen partial pressure is reduced by electrochemical oxygen pumping [7], or by metals like zirconium [8] and magnesium [9]. During reduction, the color changes from white to yellow or 
for stronger reduction to grey, and the coloration increases with time and temperature $[6,9]$ due to electrons trapped by impurities and excess oxide vacancies at energy levels closer to the conduction band in F-centers [8]. Stronger and more controlled, but localized, reduction may be obtained by cathodic polarization of an inert electrode positioned on YSZ. Following the reduction, the conductivity of pure zirconia increases by several orders of magnitude due to the formation of $n$-type charge carriers $[6,10]$, and to a certain degree this is also found for YSZ [7, 11].

When an electronic conductivity is induced in the electrolyte at a polarized electrode, the electrode reaction may spread from an initially narrow triple phase boundary (TPB) zone over the gas/electrolyte interface, thus increasing the effective reaction area [12].

The YSZ blackening at polarized electrodes has been studied optically $[1,13]$ and shows the reduction zone to spread quickly from the cathode as a tongue shaped front into the zirconia. The blackening of zirconia does not affect the lattice parameters [9, 14]; however, the blackened zirconia may in some respects behave as an inhomogeneous material [14].

Luerssen et al. [15] performed in situ studies of strong cathodic polarization of patterned Pt electrodes on polycrystalline as well as single crystal YSZ with photoelectron emission microscopy (PEEM) and scanning photoelectron microscopy (SPEM) at $350-400^{\circ} \mathrm{C}$ and observed a reduction zone moving at a rate of a few microns per second. It was shown that the reduction front advances almost like a phase transition, where electrons with energies close to the Fermi level of the polarizing electrode were emitted from the reduced phase, indicating a high electronic conductivity. X-ray photoelectron spectroscopy (XPS) showed a substantial decrease in the intensity of the $\mathrm{Zr}^{4+}$ ions and formation of lower oxidation states. The transformation from an electronic insulator to an almost metallic YSZ phase moving as a front has recently been confirmed with energy-resolved PEEM studies by Siegel et al. [16]. They found that when stepping from the native YSZ into the reduced YSZ phase there was a sudden jump in the edge of the density of states from higher to lower binding energies, and a Fermi level aligning that of the Pt electrode. Based on the time for reoxidation of the electronic conducting YSZ to the ionic conducting YSZ after exposure to an oxygen pressure of $210^{-7} \mathrm{Torr}$, they concluded that the metallic phase was confined to a 20 $\mu \mathrm{m}$ wide layer with a thickness less than $900 \mathrm{~nm}$.

In a recent work [17], Ni probe electrodes polarized on $\mathrm{YSZ}$ in a $\mathrm{H}_{2} \mathrm{O} / \mathrm{H}_{2} / \mathrm{N}_{2}$ atmosphere at $650^{\circ} \mathrm{C}$ were studied by impedance measurements and cyclic voltammetry. The main results were that the high frequency resistance decreased with one to three orders of magnitude when the polarization was increased from 0 to $-2 \mathrm{~V}$ and, similarly, that the low frequency resistance decreased with four to five orders of magnitude. The results were interpreted as caused by an increase of the electron concentration in the YSZ, changing it from a purely ionic conductor to a mainly electronic conductor. This change is accompanied by a spreading of the electrochemical $\mathrm{H}_{2} \mathrm{O}$ reduction on the YSZ|gas interface.

The aim of the present work is to improve the understanding of the processes in YSZ when in contact with a strongly cathodically polarized electrode. It is known that formation of intermetallic compounds occurs on strongly polarized Ni electrodes on YSZ [3] modifying the microstructure and chemistry of the interface. It is the experience of the present authors that, although Pt electrodes may also form intermetallic compounds with Zr [18], the process in the YSZ is much slower for Pt than for Ni electrodes. Therefore, PtIr probe electrodes were chosen for this micro scale investigation of the formation of conductive regions at cathodically polarized electrodes on YSZ by scanning probe measurements of local conductance [17] combined with impedance measurements performed with the PtIr probe as the working electrode. 


\section{Materials and methods}

\section{Materials}

Polycrystalline $8 \mathrm{~mol} \%$ yttria-stabilized zirconia was used as the electrolyte. The YSZ powder (Tosoh, TZ8Y) was pressed to disks and sintered for $2 \mathrm{~h}$ at $1500^{\circ} \mathrm{C}$ in air giving a final diameter of $1 \mathrm{~cm}$ and a thickness of $1 \mathrm{~mm}$. The disks were polished ending with a $0.1 \mu \mathrm{m}$ diamond suspension, and finally they were thoroughly cleaned. The Tosoh TZ8Y powder typically has a Si content of $10-20$ ppm as measured with glow discharge mass spectrometry.

The probe material is a PtIr alloy (Goodfellow), Pt80-Ir20, and was chosen because it has a high conductivity and is chemically and mechanically stable at high temperatures and in oxidizing as well as reducing atmospheres. The probes are fabricated in-house [19] from a $100 \mu \mathrm{m}$ thick wire which has a Si content $<50$ ppm.

\section{Methods}

Multiple polarization experiments were performed in a controlled atmosphere high-temperature scanning probe microscope (CAHT-SPM) [20, 21]. The CAHT-SPM works as a normal atomic force microscope with a fine probe and a feedback system keeping a constant force between the probe and the surface. The probe may either scan the surface to provide lateral information or be used for stationary measurements, such as local impedance spectroscopy. The microscope works in the temperature range of $25-850{ }^{\circ} \mathrm{C}$ and in dry and humidified atmospheres such as air, $\mathrm{N}_{2}$ and $9 \% \mathrm{H}_{2} / \mathrm{N}_{2}$. A potentiostat is connected to the microscope and is used for all electrochemical treatments and measurements to record the immediate effect of potential, time, and contact force.

The YSZ disks were mounted on the CAHT-SPM hot stage (Fig. 1) and fixed with platinum springs. In the polarization experiments, the furnace was heated to $900^{\circ} \mathrm{C}$ at $20^{\circ} \mathrm{C} / \mathrm{min}$ resulting in a surface temperature of $650^{\circ} \mathrm{C}$ as measured by a Pt-PtRh thermocouple in a separate temperature calibration experiment. The polarizations were carried out in an atmosphere consisting of $9 \% \mathrm{H}_{2}$ in $\mathrm{N}_{2}$ humidified by bubbling the gas through water at room temperature. The oxygen partial pressure in the chamber was measured by a $\mathrm{pO}_{2}$ monitor connected to the gas outlet to ensure that there were no significant leaks of air into the system. During polarizations the contact force was around 3-5 times the force used during scanning to ensure a good PtIr probe-YSZ contact. 


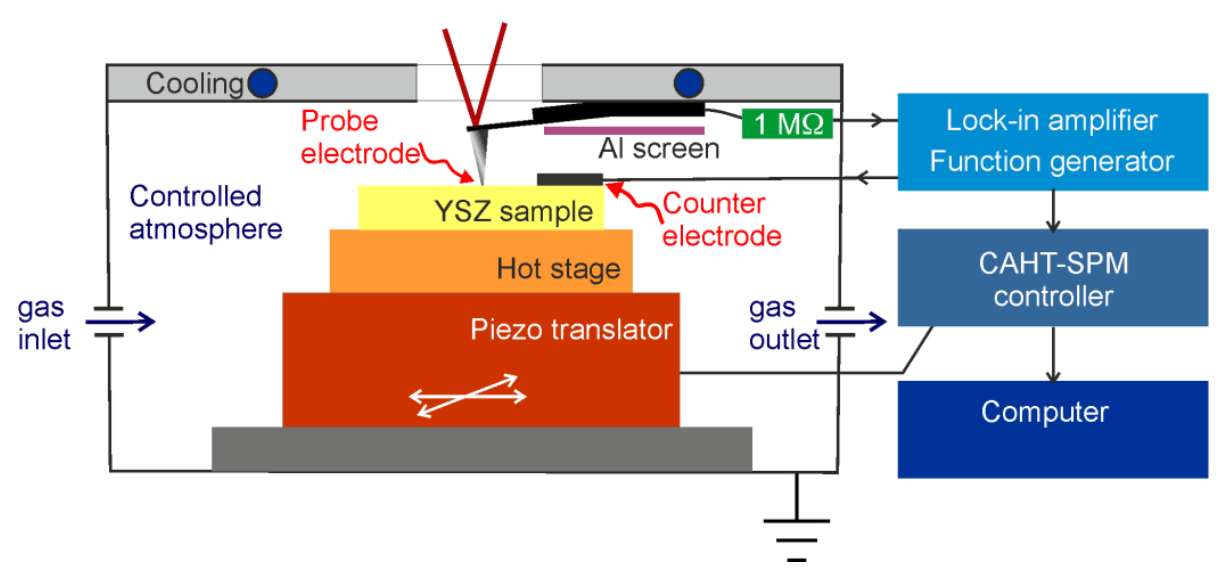

Fig. 1 Sketch of the set-up in the chamber of the CAHT-SPM

\section{Temperature}

The stated temperature refers to the temperature as measured with a thermocouple positioned on the YSZ surface. As recently pointed out by Huber et al. [22], this temperature may deviate substantially from the actual surface temperature due to heat conduction through the metallic wires. Depending on the thickness of the leads and the contact area size, the temperature at the contact area may easily be more than $100{ }^{\circ} \mathrm{C}$ lower than the actual surface temperature away from the contact point. Thus, when working with microelectrodes, as in this case where a sharp metallic probe is positioned directly on the electrolyte surface, a cooled zone that - depending on the thermal conductivity of the electrolyte - extends a number of contact radii away from the contacted area is formed. As also pointed out by Huber et al. [22], this means that the effective temperature for a process studied depends on the extension of the reaction zone. In the case of a triple phase boundary (TPB) reaction on a microelectrode with a large diameter compared to that of the probe contact, the effective temperature is the actual surface temperature, whereas for a process distributed over the entire electrode area it is a weighted average over the electrode. In the present work, where, as discussed below, the reaction zone is close to the probe-electrolyte contact at low polarizations, but at increased polarizations is widened and takes place in a non-isothermal region, the question of the reaction temperature is complicated. Thus, while a semi quantitative interpretation of the results is possible, a detailed quantitative description will be very complicated and beyond the scope of this paper.

\section{Conductance mapping}

The conductance of the sample surfaces after polarizations lasting 6-30 minutes was determined by scanning the sample with an $\mathrm{AC}$ polarized probe and measuring the resulting current. An amplitude of $0.5 \mathrm{~V}$ rms at $10 \mathrm{kHz}$ was supplied by a Stanford Research Systems SR830 Lock-In amplifier. The current through the probe was determined by the amplifier with a series resistance inserted to protect the input from overload. Conductance images were acquired simultaneously with topographic images. More details are given in [23]. 
Scanning electron microscopy (SEM) was performed with a Zeiss Merlin field emission scanning electron microscope at low accelerations voltage on non-coated samples. Energy-dispersive X-ray spectroscopy (EDS) was performed on probes and carbon-coated YSZ surfaces with a Bruker Quantax system.

Impedance spectroscopy

Electrochemical impedance spectroscopy was carried out using a Gamry Instruments FAS2 Femtostat. Because of the large area of the counter electrode compared to that of the working electrode, the counter electrode was assumed to be in equilibrium with the atmosphere and was also used as reference electrode. From thermodynamic data [24] the equilibrium potential in the $9 \% \mathrm{H}_{2} / 3 \% \mathrm{H}_{2} \mathrm{O} / \mathrm{N}_{2}$ was calculated to $-1.06 \mathrm{~V}$ relative to the potential, $E^{\ominus}\left(\mathrm{O}_{2}\right)$, of a standard oxygen electrode with $p \mathrm{O}_{2}=1$ bar at $650{ }^{\circ} \mathrm{C}$. The impedance measurements were performed in the frequency range $82 \mathrm{kHz}-0.08 \mathrm{~Hz}$ with an amplitude of $20 \mathrm{mV} \mathrm{rms}$.

Series of impedance measurements where the DC potential was stepped down or up in the range 0 to $-2 \mathrm{~V}$, and in one series from $-2 \mathrm{~V}$ to $0.5 \mathrm{~V}$, in steps of 0.05 or $0.1 \mathrm{~V}$ were made. After each step, the probe electrode was conditioned for $300 \mathrm{~s}$ before the impedance was recorded.

The same initial conditions, i.e. new sample and new probe, were attempted in the experiments but in some cases an experimental series was initiated and interrupted later because of an unstable probe-sample contact. In that case, the probe was moved to a new position before the experiment was reinitialized. Consequently, the probes may, in addition to differences in size and geometry from the fabrication, have a somewhat different history.

\section{Results}

The impedance measurements gave spectra with inductive features quite different from what is usually obtained on electrodes polarized on YSZ. Characteristic features from two series of impedance spectra, where the potential has been stepped down from the equilibrium potential, are shown in Fig. 2 and Fig. 3. At first, the spectra in the two figures look somewhat different, but a closer inspection reveals a common sequence of elements.

Close to equilibrium a very suppressed arc is seen. At potentials around -2.3 to $-2.4 \mathrm{Vvs} . E^{\ominus}\left(\mathrm{O}_{2}\right)$, the $\operatorname{arc}$ splits into two separate capacitive arcs marked C1 and C2.

After a further decrease in potential, an inductive loop, L1, appears between the capacitive arcs (Fig. 2C). The characteristic frequencies for the arcs in Fig. 3 are generally higher than those in Fig. 2, and apparently the capacitive high frequency arc, $\mathrm{C} 1$, has moved beyond the experimental frequency range in Fig. $3 \mathrm{c}$ where only the inductive loop, L1, followed by the low frequency arc, C2, are seen. In Fig. $2 \mathrm{~d}$ and $3 \mathrm{~d}$ the relative size of the inductive loop, L1, has increased, and in Fig. 3d, an inductive loop, L2, has emerged at frequencies below those of the capacitive arc, $\mathrm{C} 2$. It is noted that, because of the lowering of the characteristic frequency of L1 from Fig. 3c to Fig. 3d, the inductive loops and the capacitive arc, C2, now overlap and C2 is drawn into the inductive region. Most likely, L2 would also have been observed in Fig. $2 \mathrm{~d}$ if the frequency range had included lower frequencies. At still lower potentials, the relative size of the 
inductive high frequency loop, L1, decreases (Fig. 2e) and subsequently it vanishes as seen in Fig. $2 \mathrm{f}$.

Summarizing the impedance measurements, the general picture - going from high to low frequencies - is a capacitive arc followed by a sequence of an inductive, a capacitive and an inductive arc. Depending on the polarization, some of the arcs may be missing or are located or beyond the experimental frequency range. The inductive behavior at high frequencies is found in spectra recorded for decreasing as well as increasing frequency and potential. The inductive loops are, thus, not due to drift in the system during the measurements. Since the measurements are performed in a two electrode setup with a very small working electrode against a large combined counter-reference electrode, artifacts caused by electrode crosstalk [25] and misalignment [26] can also be ruled out.
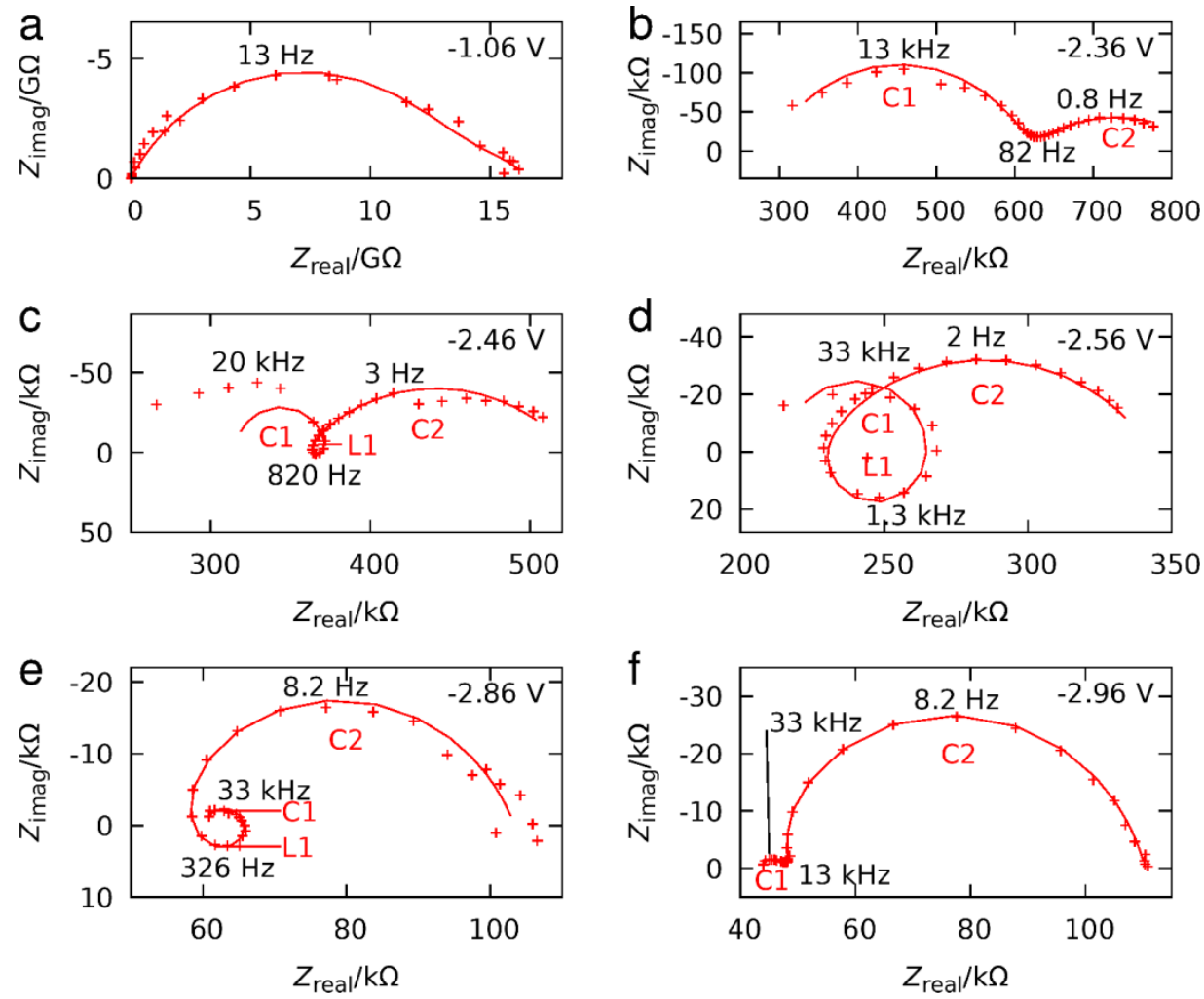

Fig. 2 Impedance in the frequency range $82 \mathrm{kHz}-0.08 \mathrm{~Hz}$ recorded when stepping the electrode polarization from $0 \mathrm{~V}$ to $-1.9 \mathrm{~V}$. a) $0 \mathrm{~V}, \mathrm{~b})-1.3 \mathrm{~V}, \mathrm{c})-1.4 \mathrm{~V}$, d) $-1.5 \mathrm{~V}, \mathrm{e})-1.8 \mathrm{~V}$ and f) $-1.9 \mathrm{~V}$. The full line shows a fit to the equivalent circuit shown in Fig. 10, and the frequencies correspond to the local maxima and minima for $Z_{\text {imag. }}$. The potential in the upper right corner of the graphs is the potential of the polarized electrode relative to $E^{\theta}\left(\mathrm{O}_{2}\right)$. The probe used in this experiment had been polarized to $-2 \mathrm{~V}$ for $3 \mathrm{~h}$ on a different position on the same sample in a previous unsuccessful experiment 

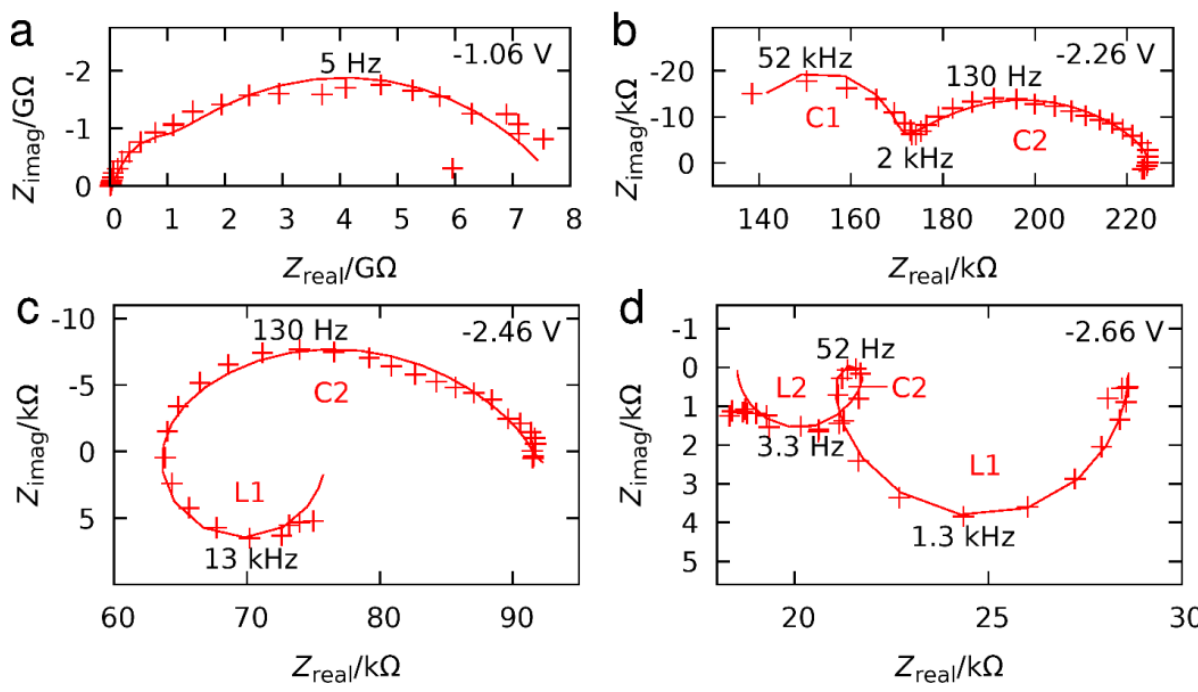

Fig. 3 Impedance in the frequency range $82 \mathrm{kHz}-0.08 \mathrm{~Hz}$ recorded when stepping the electrode polarization from $0 \mathrm{~V}$ to $-1.6 \mathrm{~V}$. a) $0 \mathrm{~V}, \mathrm{~b})-1.2 \mathrm{~V}, \mathrm{c})-1.4 \mathrm{~V}$ and d) $-1.6 \mathrm{~V}$. The full line shows a fit to the equivalent circuit shown in Fig. 10, and the frequencies correspond to the local maxima and minima for $Z_{\text {imag. }}$. The potential in the upper right corner of the graphs is the potential of the polarized electrode relative to $E^{\ominus}\left(\mathrm{O}_{2}\right)$. This experiment was made with a probe and a sample that had not been used before

Conductance mapping of the area around the location where the probe was situated during the polarization was carried out after the polarization was released, and characteristic spots were found. The spots may show some or all of several features. The most common feature is a highly conducting area with the size of a few microns (Fig. $4 a$ and b). The conducting area has been observed for polarizations between $-0.8 \mathrm{~V}$ and $-2 \mathrm{~V}$ for polarizations as short as $30 \mathrm{~s}$, and after the longest polarizations lasting a few hours. One or more low conductance regions are usually observed next to the high conducting area and/or a little distance away from it (Fig. 4b, Fig. 5b). The conductance in these areas is clearly much lower than for the YSZ. Some polarizations, mainly the longer ones, result in addition to the spot in the conductance map, also in the formation of an elevated area in the topography image (Fig. 5a). This particular elevated area is 80 $\mathrm{nm}$ higher than the surroundings. The high conductance spots and the spots in the topographic maps appear in subsequent scans, and they are persistent after cooling, waiting for $24 \mathrm{~h}$ and then reheating (Fig. $6 a \mathrm{og}$ b). In some cases, for both short and long polarizations, a much larger affected area is seen. It is observed as a circular area with a radius many times the contact area radius (Fig. 4a). The area has a higher conductance than the surrounding YSZ. 

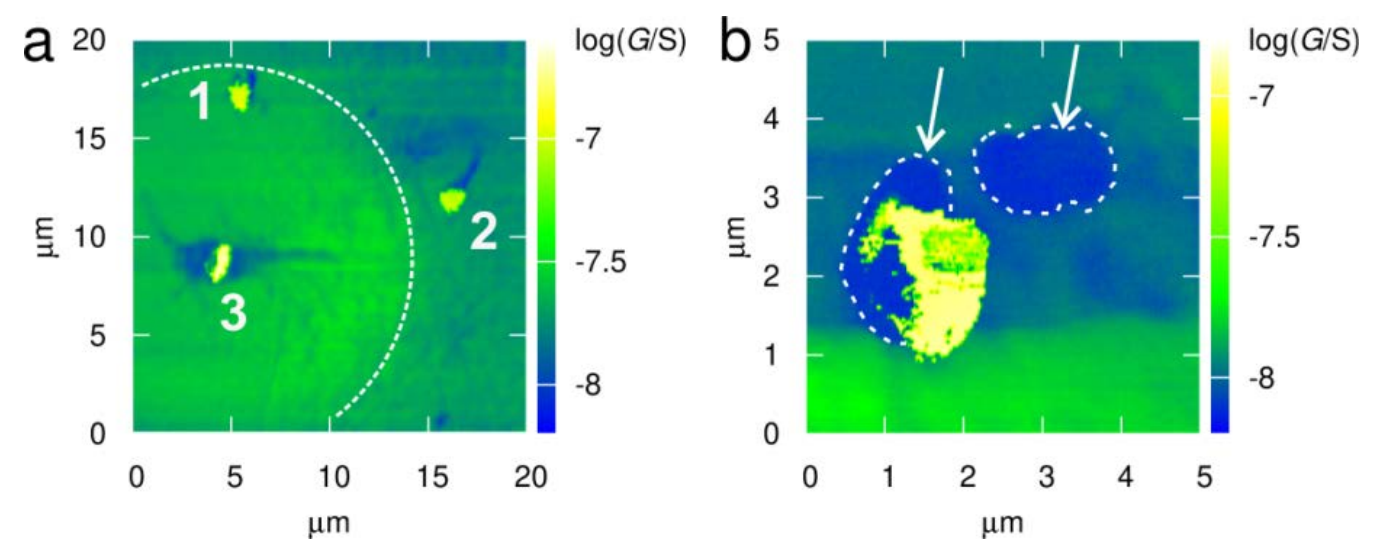

Fig. 4 Conductance maps obtained at $650{ }^{\circ} \mathrm{C}$ showing a) three high conductance spots (yellow) resulting from three subsequent polarizations ( $1:-1.5 \mathrm{~V}$ for $360 \mathrm{~s}, 2:-1.5 \mathrm{~V}$ for $600 \mathrm{~s}, 3:-2 \mathrm{~V}$ for $360 \mathrm{~s}$ ). For the last obtained spot ( $-2 \mathrm{~V}$ for $360 \mathrm{~s}$ ) a circular area with a diameter of $\sim 20 \mu \mathrm{m}$ is observed (white dotted line) where the conductance is higher than for the YSZ. b) A more detailed scan of a high conducting region (-2 V for $300 \mathrm{~s}$ ) and at least two low conducting regions (arrows and dotted lines).
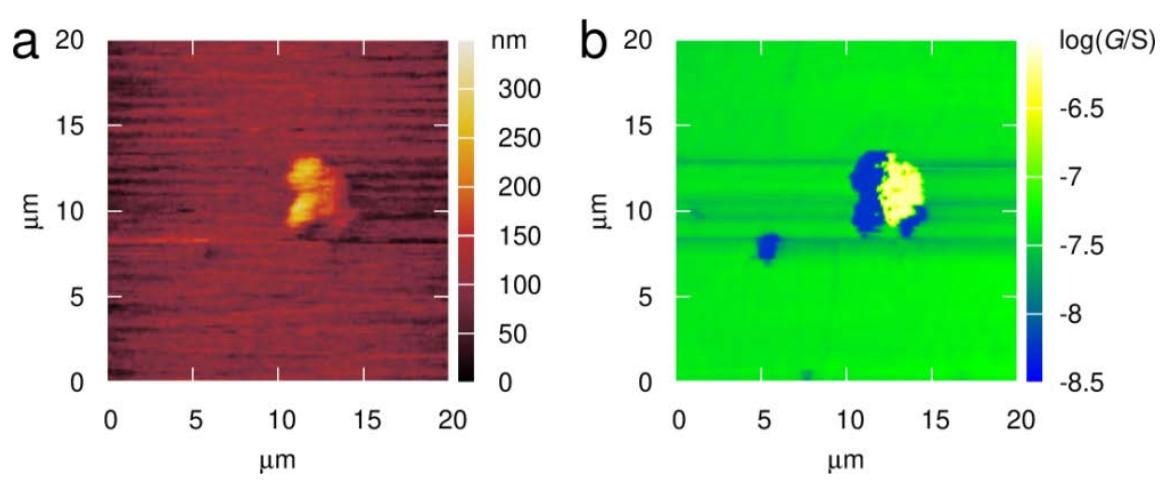

Fig. 5 a) Topography image of a spot after a polarization of $-1.5 \mathrm{~V}$ for 30 minutes. b) Conductance map of the same area. There is a clear high conductance region and two or three low conducting areas. Both images were obtained at $650^{\circ} \mathrm{C}$. 

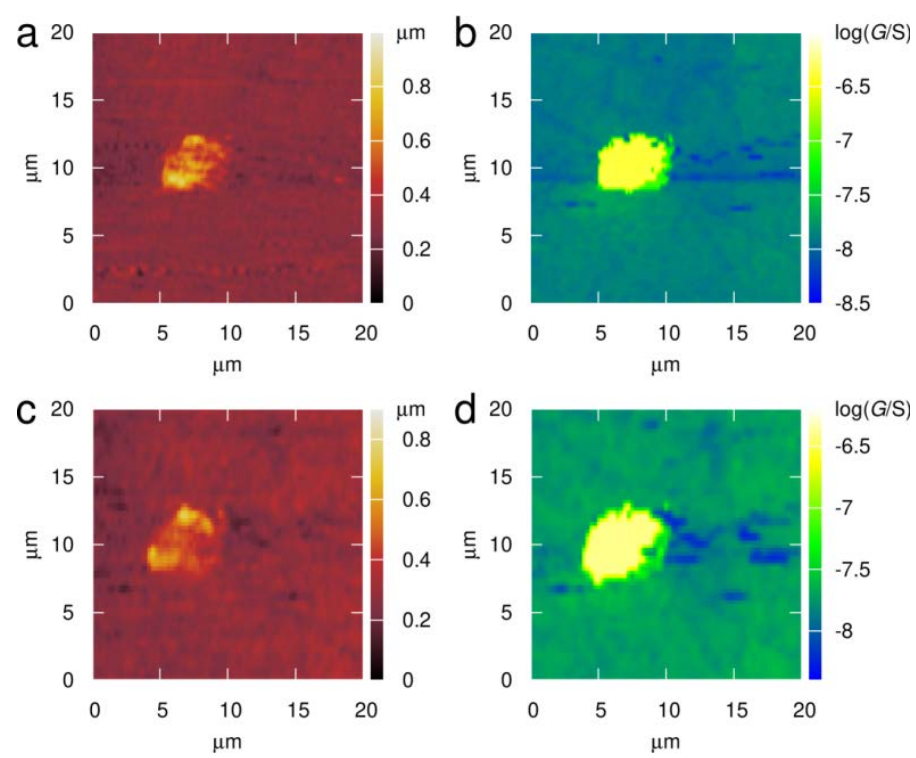

Fig. $6 \mathrm{a}, \mathrm{b})$ Topography and conductance maps after a polarization of $-2 \mathrm{~V}$ for $600 \mathrm{~s}$, obtained at $650^{\circ} \mathrm{C}$. c, d) Topography and conductance of the same spot obtained the next day after staying at room temperature for the night and reheating to $650^{\circ} \mathrm{C}$

In the few cases where it was possible to locate the contact areas on the YSZ, it is clear that there are both microstructural and chemical changes to the contact area and surroundings. In general, we find that the contact areas and their surroundings are associated with the presence of silicate. In a few experiments where a high current was observed during polarization, the contacts points can easily be identified, and for one sample an area $>100 \mu \mathrm{m}$ in diameter is dotted with silicate particles that are a few microns in size. The silicate particles are rounded and wetting the YSZ as if they have been fluid. Na and K are associated with the contact areas and the silicate phase. EDS performed on the probe part of the contact areas shows that minor amounts of $\mathrm{SiO}_{2}$ and $\mathrm{Al}_{2} \mathrm{O}_{3}$ are present, and the probe sides close to the contact may be covered with spherical Al and Si containing particles with sizes below $500 \mathrm{~nm}$.

\section{Discussion}

The high frequency resistance of the probe|YSZ contact, $R^{\infty}$, is determined by extrapolating the high frequency impedance data to the "infinite" frequency value on the real axis. At polarizations of less than about $-0.5 \mathrm{~V}$ where the impedance spectra range from a few $\mathrm{M} \Omega$ at high frequencies to $\sim 10 \mathrm{G} \Omega$ at low frequencies, the uncertainty in the high frequency extrapolation may be up to $50 \%$, and the results may be influenced by stray capacitance ( $1 \mathrm{pF}$ stray capacitance will give a shunting impedance of $1.6 \mathrm{M} \Omega$ at 100 $\mathrm{kHz}$ ). At lower potentials the extrapolations are more accurate ( $<5 \%$ at $-2 \mathrm{~V}$ polarization). As seen from Fig. $7 \mathrm{a}, R^{\infty}$ decreases almost three orders of magnitude when the potential is stepped downwards from the equilibrium potential, $-1.06 \mathrm{~V}$ vs. $E^{\ominus}\left(\mathrm{O}_{2}\right)$ to $-3.06 \mathrm{~V}$ vs. $E^{\ominus}\left(\mathrm{O}_{2}\right)$, i.e. a polarization of $-2 \mathrm{~V}$. 

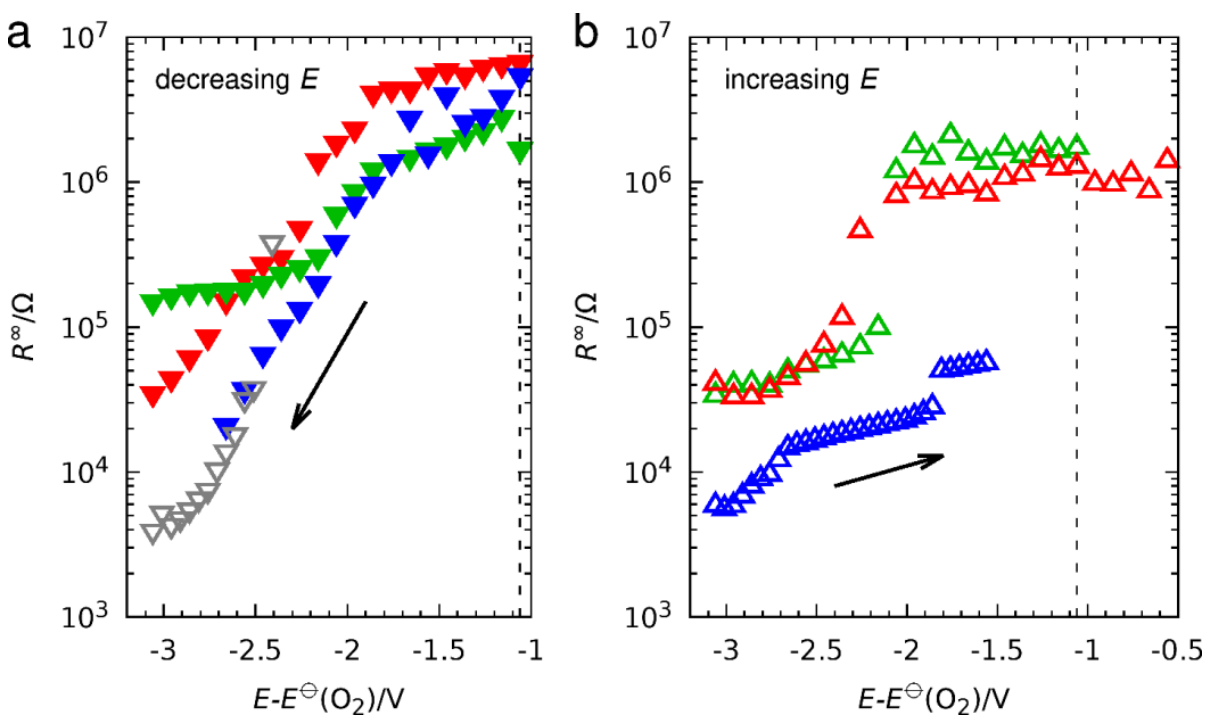

Fig. $7 \mathrm{High}$ frequency resistance, $R^{\infty}$, as function of the electrode potential relative to the standard oxygen electrode potential. Except for the blue symbols, each series in a) and b) is recorded on a new YSZ disc with a new probe. The dotted vertical lines at $-1.06 \mathrm{~V}$ indicate the equilibrium potential for the probe electrode in the present atmosphere. a) $R^{\infty}$ for cathodic polarizations increasing from 0 to $-2 \mathrm{~V}$. The red symbols correspond to the impedance series in Fig. 2 and the blue symbols to that in Fig. 3. b) $R^{\infty}$ for polarizations decreasing from -2 to $+0.5 \mathrm{~V}$. The open blue symbols show a series recorded a few days later on the sample also used for the blue curve in a).

Correspondingly, an increase in the high frequency resistance is seen when the potential is stepped in the positive direction as shown in Fig. 7b. However, the increase is less than two orders of magnitude, i.e. significantly smaller than the decrease obtained for downwards potential stepping and shows a rather abrupt transition around $-2 \mathrm{~V}$ followed by a plateau that extends even into to the anodic region (open red symbols in Fig. 7b). The hysteresis could indicate that a true steady state has not been obtained, but the length of the plateau ( $\sim \mathrm{h}$ for the open red symbols) shows that the relaxation processes - if present have time constants large compared to the upwards stepping rate. The relaxation is therefore too slow to be investigated with the present equipment due to drift of the probe position. Also, the conductance maps in Fig. 6b) and d) show that the changes that were introduced are decaying very slowly. In previous works, the conductance change is reported to be reversible in air [27], and even strongly reduced samples can be reoxidized to their pristine state in oxygen [11]. The samples in the present work have only been exposed to the humidified hydrogen when heated, and may therefore not be completely reoxidized. The results shown in Fig. 7 and Fig. 9 are similar to those found for the Ni|YSZ interface [17], although the enhanced conductivity induced in the YSZ seems more persistent to reoxidation in the Pt|YSZ system.

It is well known that at very low oxygen partial pressures or - equivalently - negative polarizations [7] electronic conductivity develops in YSZ electrolytes [28]. The increase in conductivity is attributed to a partial reduction of zirconia. Thus, Fig. 7a most likely shows how the YSZ electrolyte changes from an ionic conductor to a mainly electronic conducting material. This is consistent with the highly conducting spots observed after release of the polarization (Fig. 4). 
The radius of the volume where the conductivity of YSZ has been increased can be crudely estimated by assuming that a hemisphere with a conductivity much higher than that of unpolarized YSZ has grown from the probe|YSZ interface into the YSZ. The high frequency resistance is then determined by the YSZ outside the hemisphere and is - due to the spherical symmetry - inversely proportional to its radius. To account for the decrease in $R^{\infty}$ of more than two orders of magnitude, the radius of the hemisphere formed at the strongest polarization is more than 100 times larger than the radius of the physical probe|YSZ contact. For the impedance series depicted in Fig. 2 and Fig. 3, the high frequency resistance without polarization is around $5 \mathrm{M} \Omega$ and $2 \mathrm{M} \Omega$, respectively. Using a conductivity of $9.710^{-3} \mathrm{~S} \mathrm{~cm}^{-1}$ [29] the corresponding probeYSZ contact radii are calculated to $r=\frac{1}{4 \times R^{\infty} \times \sigma}=\frac{1}{4 \times 510^{6} \times 9.710^{-3}} \mathrm{~cm} \approx 50 \mathrm{~nm}[30,31]$ for the red curve and $125 \mathrm{~nm}$ for the blue curve. The decrease of $R^{\infty}$ thus requires a region of enhanced conductivity that extends more than $5 \mu \mathrm{m}$ from the physical probe contact point, and has a size similar to that of the highly conductive spots in Fig. 4 and Fig. 5. Comparing the red and blue curves in Fig. 7a, there is almost an order of magnitude difference between the resistance values at $-2.6 \mathrm{~V}$ vs. $E^{\circ}\left(\mathrm{O}_{2}\right)$. At this potential, the $\mathrm{DC}$ current for the red curve is $-1 \mu \mathrm{A}$, whereas it is $-10 \mu \mathrm{A}$ for the blue curve, i.e. the ratio between the $\mathrm{DC}$ reaction rates is close to the ratio of the reciprocal high frequency resistances. The difference could be interpreted as a consequence of local heating caused by the irreversible electrode and transport processes close to the probe-YSZ contact. However, for the ionic conductivity to increase an order of magnitude the temperature several microns away from the probe contact has to be raised by $\sim 100{ }^{\circ} \mathrm{C}$, which appears unlikely. Accepting that the decrease of $R^{\infty}$ with increasing negative polarization is mainly due to an enhanced electronic conductivity of the YSZ, the overall picture is as sketched in Fig. 8. When the probe electrode is polarized to negative potentials the concentration of $n^{\prime}$ species at the probe|YSZ interface is increased by the electrochemical reaction

$$
\mathrm{e}^{-}(\mathrm{Pt}) \rightleftarrows \mathrm{n}^{\prime}(\mathrm{YSZ})
$$

where $\mathrm{n}^{\prime}$ may represent $\mathrm{Zr}^{+4}$ ions reduced to $\mathrm{Zr}^{+3}$ or even $\mathrm{Zr}^{+1}[15,9]$ or electrons in a metallic band structure close to the Fermi level of the polarized electrode $[15,16]$.

Direct electronic conduction from cathode to anode has been reported for planar SOEC cells at $810^{\circ} \mathrm{C}$ [32] where a limiting cell voltage close to $1.9 \mathrm{~V}$ was observed and it was possible to operate the cell at a stable potential at $0.34 \mathrm{~A} \mathrm{~cm}^{-1}$ for $64 \mathrm{~h}$ without any supply of water. In another experiment, the $\mathrm{Ni}$ anode of SOFC cells was activated at $\sim 700^{\circ} \mathrm{C}$ by reverse current pulses from 0.25 to $6 \mathrm{~A} \mathrm{~cm}^{-1}$ and varying water content in the fuel gas [33]. The fact that a limiting current was not observed, and that a constant cell voltage was obtained for pulse currents exceeding what could be provided by the water in the fuel gas was taken as supporting evidence for the introduction of electronic conductivity in the YSZ electrolyte. These investigations were carried out on cells with large electrodes $\left(45 \mathrm{~cm}^{2}\right.$ [32] and $1 \mathrm{~cm}^{2}$ [33], respectively) on very thin $(5-20 \mu \mathrm{m})$ electrolytes. The current field is therefore, except for minor constrictions, perpendicular to the electrolyte, and the electronic conductivity spreads as a front from the cathode towards the positively polarized anode where a $n-p$ junction is established [34].

The planar thin electrolyte geometry is very different from the geometry in the present work where the electrode is very small, up to a few microns, compared to the thickness of the electrolyte ( $1 \mathrm{~mm})$. The current field at high frequencies is therefore close to being radial, and the AC overvoltage mainly reflects processes close to the probe contact. At the transition from the highly electronic conducting region (the red volume in Fig. $8 \mathrm{a}$ and b) to the blue region where the electronic conductivity is negligible compared to the 
ionic, the flux of electrons is capacitively coupled to a current carried by oxide ions [35]. When the frequency is lowered this coupling weakens, and the impedance contribution from this charge transport route increases. The increase favors the alternative route where the electronic charge transport is coupled to the ionic via reduction of water (reaction 2) at the YSZ|gas interface of the electronic conducting region, and a steady state can be achieved with ionic conductivity and without any electronic conductivity at the counter electrode. At moderate polarizations, the reaction zone is confined to a narrow region close to the physical triple phase contact, but with increasing polarization the reaction zone widens as shown in Fig. 8 b.

$$
2 \mathrm{n}^{\prime}+\mathrm{V}_{\mathrm{O}}^{\bullet \bullet}+\mathrm{H}_{2} \mathrm{O}(\mathrm{g}) \rightleftarrows \mathrm{O}_{\mathrm{O}}+\mathrm{H}_{2}(\mathrm{~g})
$$

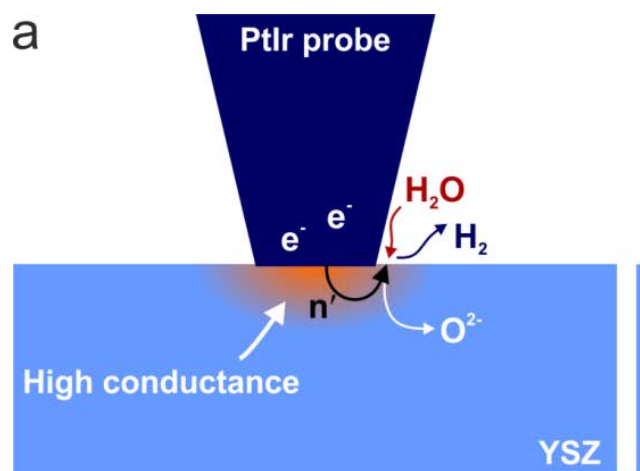

Charge transfer and migration into electrolyte

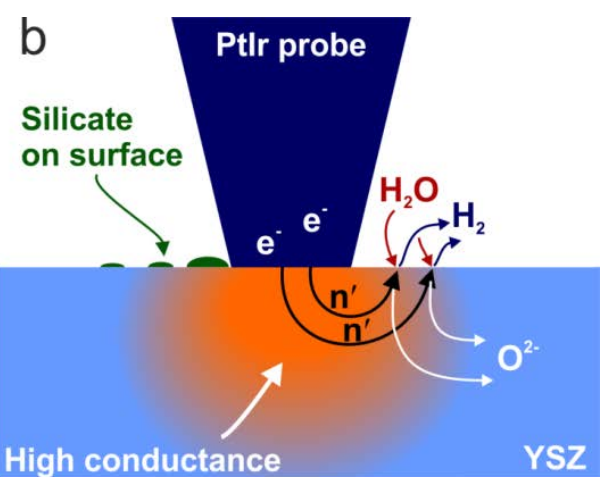

YSZ | gas reaction

Fig. 8. Sketch of the probe /YSZ contact area environment during polarization. a) Electrons are transferred across the PtIr|YSZ interface, migrate into the YSZ phase and create an enhanced conductivity region that spreads out with increasing polarization. b) Reduction of $\mathrm{H}_{2} \mathrm{O}$ at the expanding YSZ /gas interface 

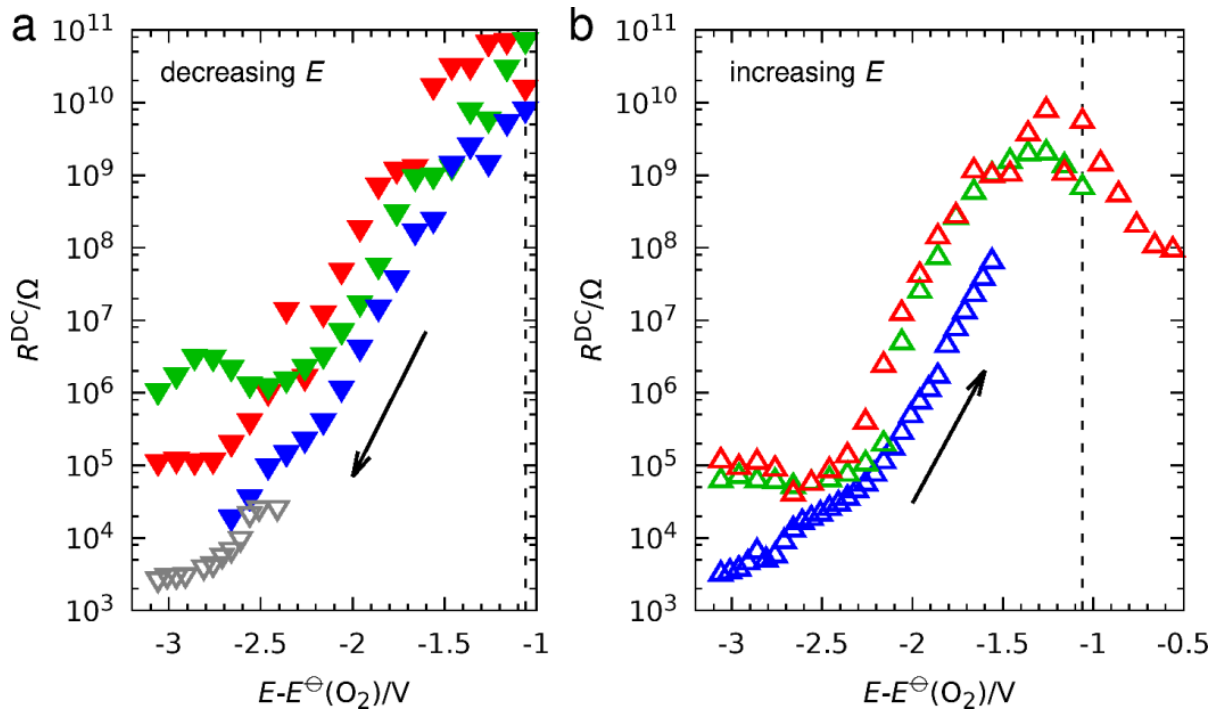

Fig. $9 \mathrm{DC}$ resistance, $R^{\mathrm{DC}}$, as function of the electrode potential relative to the standard oxygen electrode potential. Except for the blue symbols, each series in a) and b) is recorded on a new YSZ disc with a new probe. The dotted vertical lines at $-1.06 \mathrm{~V}$ indicate the equilibrium potential for the probe electrode in the present atmosphere. a) $R^{\mathrm{DC}}$ for cathodic polarizations increasing from 0 to $-2 \mathrm{~V}$. The red symbols correspond to the impedance series in Fig. 2 and the blue symbols to that in Fig. 3. b) $R^{\mathrm{DC}}$ for polarizations decreasing from -2 to $+0.5 \mathrm{~V}$. The open blue symbols show a series recorded a few days later on the sample also used for the blue curve in a).

To illustrate the effect of the spreading of the surface reaction zone, the $D C$ resistances, $R^{\mathrm{DC}}$, determined as the low frequency intercept of the impedance and the real axis for increasing as well as decreasing polarizations are shown in Fig. 9. It is noted that $R^{\mathrm{DC}}$ includes the high frequency ohmic resistance, $R^{\infty}$ as well as contributions from the electrode reaction, mass transfer, and the reaction at the YSZ|gas interface. Similar to the high frequency resistance and results obtained for Ni|YSZ [17], the DC resistance decreases with increasing cathodic polarization. The decrease is close to six decades compared to three decades for $R^{\infty}$. The difference can be explained by the fact that $R^{\infty}$ from a sphere embedded in a medium with lower conductivity is inversely proportional to the radius of the sphere, whereas $R^{\mathrm{DC}}$ is inversely proportional to the area of the surface reaction zone, i.e. to the square of the radius of the zone, if this is large compared to that of the probe /YSZ contact area. In contrast to the high frequency resistance, the DC resistance for increasing potential shown in Fig. 9b is fairly close to that determined for downwards stepping (Fig. 9a), although the final value seems slightly lower than the value before polarization. When the potential is stepped into the anodic region (the open red symbols in Fig. 9b), the DC resistance shows a maximum slightly below the equilibrium potential. At these low polarizations the electrode reaction is concentrated close to the physical triple phase boundary, and most likely controlled by the kinetics of the oxidation/reduction reactions. If the reaction rate follows the Butler-Volmer equation, a maximum would be expected near the equilibrium at a potential determined by the symmetry factor of the electrochemical reaction.

The impedance plots in Fig. 2 and Fig. 3 fall within three potential regions. Above $-1 \mathrm{~V}$ a single depressed arc is obtained. At stronger cathodic polarizations, the arc separates into two arcs, C1 and C2 (Fig. 2b and Fig. 3b). Polarizing further, the high frequency arc, C1, is followed by an inductive loop, L1, separating the 
two capacity arcs as seen in Fig. 2c-d. It is noted that in Fig. 3d the size of the high frequency inductive loop, $\mathrm{L} 1$, exceeds that of the low frequency capacitive arc, C2, and also an inductive low frequency loop, L2, has developed, giving the remarkable result of a DC resistance lower than the high frequency resistance.

Comparing the impedance series on which Fig. 7 and Fig. 9 are based, there seems to be the trend that for the series where the lowest impedance values are determined $R^{\mathrm{DC}}$ (blue and grey symbols in Fig. 7a, $\mathrm{b}$ and Fig. 9a, b) is lower than $R^{\infty}$, whereas those with higher impedance (red and green symbols in Fig. 7a and b and Fig. 9 a and b) follow the pattern in Fig. 2. Also, the characteristic frequencies are generally higher for the low impedance series, compared to those for the series with higher impedance. Whether the difference between $R^{\infty}$ and $R^{\mathrm{DC}}$ values and the characteristic frequencies at the strongest polarizations is caused by differences in contact area size, in activation depending on the position of the probe relative to grain boundaries or other factors is an open question. At stronger polarization, the relative size of the inductive loop L1 decreases (Fig. 2d-e) and has disappeared in Fig. 2 f.

As sketched in Fig. 8, the overall reaction path contains three steps: First charge (electrons) is transferred across the probe |YSZ interface. Next, the electrons migrate to the YSZ|gas interface where they participate in the water reduction process and the electronic charge transfer is converted into an oxide ion flux to the counter electrode. Ignoring geometrical complications, the reaction can qualitatively be represented by an equivalent circuit containing three blocks in series as outlined in Fig. 10. The charge transfer block describes the combined parallel reactions of electrons transferred across the probe |YSZ interface into the YSZ phase or reacting at the TPB. These processes are represented by an $R$ - $Q$ circuit giving a depressed semicircle in the complex plane. The next step, migration of $n^{\prime}$ species through the YSZ phase to the reaction at the YSZ Igas interface, is a more complex electrodiffusion process. Due to the requirement of electroneutrality, local changes in the concentration of electrons in the YSZ imply charge compensation by oxide vacancies. Thus, electronic fluxes are coupled to vacancy fluxes and the migration processes are driven by both concentration gradients and electric fields, as described by the Nernst-Planck equation.

It is known from biophysical experiments [36] and numerical simulations [37] that in the case of a permeable membrane separating two binary electrolyte solutions with different concentrations and a common anion and cations with different mobilities, i.e. a system not in equilibrium, the impedance across the membrane shows - depending on the relative mobilities - either capacitive or inductive behavior [36, 37]. More recently, in a treatment of the impedance of an ion-exchange membrane system, a solution of the Nernst-Planck equations based on exponential integrals has been obtained for a finite diffusion layer at an ion-exchange membrane system [38]. The boundary conditions in the solution is the transfer of one specific ion from the membrane into the diffusion layer which is terminated by a constant salt concentration, i.e. similar to those of the present system, where electrons are injected into, or depleted from, YSZ. The solution shows that for the current direction where the salt concentration at the membrane surface is enriched the impedance contains an inductive contribution, whereas a capacitive response is obtained for the depleting current direction. This behavior has recently been demonstrated experimentally for a solid state system with a Fe-doped $\mathrm{SrTiO}_{3}$ film sandwiched between an oxide ion blocking electrode and a reversible oxygen electrode [39]. For positive polarization of the p-type conducting oxide, where the p-concentration inside the layer is enhanced, the oxide showed inductive behavior at low frequencies, whereas capacitive arcs were obtained at negative depleting potentials.

Although the membrane and $\mathrm{SrTiO}_{3}$ systems are different from the present, they have the common features of a steady state electrodiffusion being superimposed by an AC perturbation: At high frequency 
the steady state concentration profile is not disturbed, and the migration is determined by the electric field only. When the frequency is lowered, a damped concentration wave with a wavelength which is increasing with decreasing frequency is superimposed the steady state profile and an electric AC field develops and adds to the driving force. At very low frequency, a quasi-steady state situation develops, and the impedance is equal to the slope of the stationary overpotential-current relation. The decrease of the impedance - from the high frequency resistance to lower impedances at lower frequencies - is described by the center block in Fig. 10. The characteristic frequency of the arc, C2, in Fig. 2 is lower than that of C2 in Fig. 3 where the DC impedance is an order of magnitude lower. The lower frequency could be the result of a larger region of enhanced conductivity which requires a longer wavelength before DC conditions are reached.

The final step in the reaction sequence in Fig. 8 is the reduction of water on the YSZ|gas interface facilitated by the electronic conductivity induced in the YSZ by the cathodic polarization and causing the active reaction area to increase with increasing polarization. The $A C$ signal superimposed on the steady state causes the reaction area to oscillate. At high frequencies, this oscillation is confined to the vicinity of the probe|YSZ contact. Due to the strong stationary polarization here, the reaction rate is most likely limited by the diffusion of water to the surface, and no AC response is obtained. At lower frequencies, the oscillating active area increases and spreads outside the limiting current region, and the oscillation modulates the stationary current and yields an $\mathrm{AC}$ response. When the area, as in the present case, increases with increasing polarization, the area has its maximum at the end of the half wave with strongest polarization. Thus, the current response is lagging behind the polarization and an inductive response is obtained. It therefore seems reasonable to ascribe the low frequency inductive arc seen in Fig. $3 d$ to the gas|YSZ interface reaction and represent it with the block at the right in Fig. 10.

Although the equivalent circuit in Fig. 10 can describe all the features observed in the impedance series shown in Fig. 2 and Fig. 3 , it is only a crude modeling, ignoring the fact that the - in itself complex reaction sequence proceeds in 3-dimensional space. A more detailed analysis would require techniques like finite element modeling. Impedance spectra very similar to those in Fig. 2c-d have previously been obtained for the reduction of water on ceria-doped YSZ surfaces and ascribed to the spreading of the reaction zone from the triple phase boundary onto the YSZ | gas interface [40].

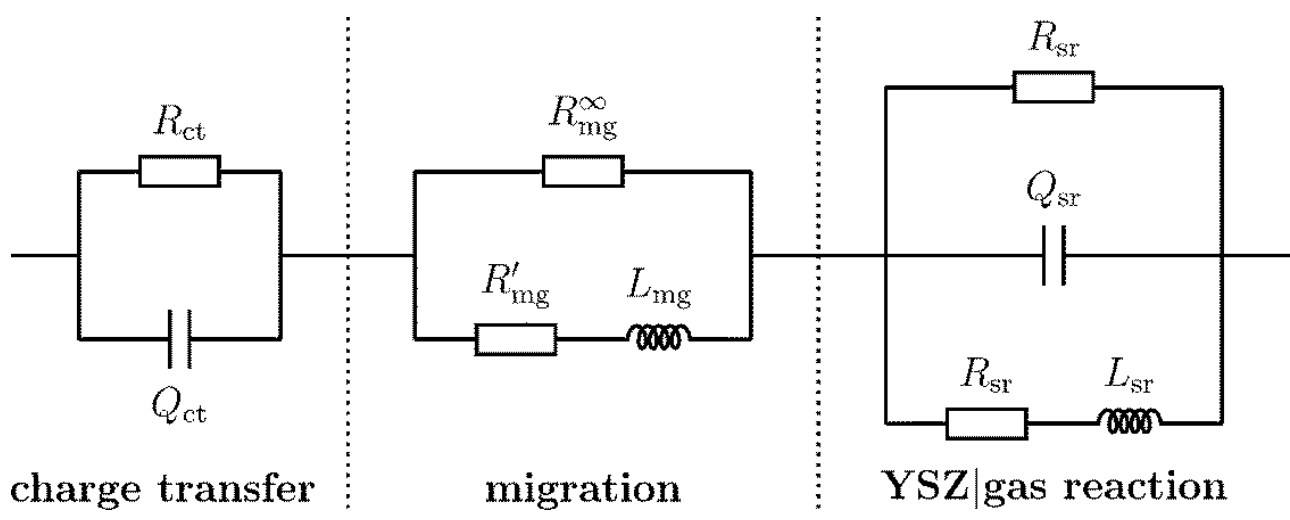

Fig. 10 Equivalent circuit for the electrode reaction as sketched in Fig. 8. The $Q$ components are capacitive constant phase elements. The charge transfer (ct) reaction at the probe|YSZ interface is described by the $R_{c t}$ - $Q_{c t}$ parallel combination. The migration branch $(\mathrm{mg})$ covers the migration of ionic as well as electronic 
charge carriers into the YSZ bulk phase, and the YSZ surface|gas reaction (sr) part describes migration of $n^{\prime}$ species to the YSZ|gas interface and the $\mathrm{H}_{2} \mathrm{O} / \mathrm{H}_{2}$ redox reaction there

The reduction potentials for $\mathrm{ZrO}_{2}$ and $\mathrm{SiO}_{2}$ (glass) relative to a standard oxygen electrode $\left(p \mathrm{O}_{2}=1\right.$ bar) at $650^{\circ} \mathrm{C}$ are calculated from thermodynamic data [24] to $-2.4 \mathrm{~V}\left(p \mathrm{O}_{2}=410^{-53} \mathrm{bar}\right)$ and $-1.9 \mathrm{~V}\left(\mathrm{pO}_{2}=1.210^{-42}\right.$ bar), respectively. In the present setup, silicon may therefore be formed at polarizations below $-0.85 \mathrm{~V}$ and zirconium below -1.4 V, or, if intermetallic Pt-Si-Zr compounds are formed, at somewhat higher potentials. As they have a high electronic conductivity, they may enlarge the effective electrode area and thus contribute to the observed decrease in electrolyte resistance. Silicate in relatively large amounts is associated with the contact area and could result from reoxidized Si containing phases. Migration of $\mathrm{Si}$ is seen in technological SOEC cathodes where it originates from silicate impurities that are reduced due to the low electrode potential and subsequently migrate into the Ni where it precipitates as nanosized silica particles [41]. The low conducting particles or areas found in the vicinity of the probe contact are most likely caused by silica from the YSZ. The YSZ typically contains $10-20 \mathrm{ppm}$ Si which is distributed in grain boundaries and triple points after sintering [42]. During thermal treatment, it migrates to the YSZ surface to form a glassy film. This is, however, a slow process at $650^{\circ} \mathrm{C}$ and it does not explain the formation of particles. The silicate migration to the contact area could be driven by a gradient in the surface tension of the film caused by the cooling effect of the probe. Another possibility is that the migration is due to the electric field close to the probe contact. The probe is also a possible source but the amount of probe material and thus the amount of Si in it is very small.

\section{Conclusion}

Local strong cathodic polarization results in significant changes in the electrical properties of YSZ, and the results in this study are in accordance with those obtained for the Ni|YSZ interface [17]. In particular, the high frequency impedance decreases almost exponentially by more than two orders of magnitude when the PtIr probe |YSZ interface is polarized with $-2 \mathrm{~V}$ in a humidified atmosphere of $9 \% \mathrm{H}_{2}$ in $\mathrm{N}_{2}$. With the cathodic polarization, the $\mathrm{H}_{2} \mathrm{O} \mid \mathrm{H}_{2}$ electrode reaction spreads from the triple phase boundary onto the YSZ |gas interface. Impedance measurements show a transition from a simple one arc spectrum to spectra with two inductive loops at strong polarization. The inductive behavior at high frequencies is ascribed to frequency dependency of the charge transport process close to the PtIr|YSZ interface, when changing from a process driven only by the oscillating electric field to a process also facilitated by the electric field created by the gradients in the damped charge carrier concentration wave, i.e. an oscillating diffusion potential. At low frequencies the YSZ / gas interface reaction area starts oscillating and combined with the DC polarization an inductive AC response is obtained. The polarization causes a local, persistent, electronic conductivity as shown by conductive spots which confirms the formation of a persistent decrease of the high frequency resistance. Silicate impurities are associated with the PtIr-YSZ contact area and migrate there during polarization.

\section{Acknowledgment}

We gratefully acknowledge financial support from Energinet.dk through the ForskEL programme "Solid Oxide Fuel Cells for the Renewable Energy Transition" contract no 2014-1-12231 and from ECoProbe (DFF - 
4005-00129) funded by the Danish Independent Research Council. Stimulating discussions with Mogens Bjerg Mogensen and Christodoulos Chatzichristodoulou are greatly appreciated.

\section{References}

1. Casselton REW (1974) J Appl Electrochem 4:25-48.

2. Chen M, Liu Y-L, Bentzen JJ, Zhang W, Sun X, Hauch A, Tao Y, Bowen JR, Hendriksen PV (2013) J Electrochem Soc 160:F883-F891.

3. Hansen KV, Chen M, Jacobsen T, Thydén K, Simonsen SB, Koch S, Mogensen M (2016) J Electrochem Soc 163:F1217-F1227.

4. Szász J, Klotz D, Störmer H, Gerthsen D, Ivers-Tiffée E (2013) ECS Trans 57:1469-1478.

5. Klotz D, Butz B, Leonide A, Hayd J, Gerthsen D, Ivers-Tiffée E (2011) J Electrochem Soc 158:B587B595.

6. Eder D, Kramer R (2002) Phys Chem Chem Phys 4:795-801.

7. Levy M, Fouletier J, Kleitz M (1988) Solid State Sci Technol 135:1584-1589.

8. Ben-Michael R, Tannhauser DS (1991) Appl Phys A 53:185-188.

9. Sinhamahapatra A, Jeon J-P, Kang J, Han B, Yu J-S (2016) Scientific Reports 6:27218.

10. Eder D, Kramer R (2006) Phys Chem Chem Phys 8:4476-4483.

11. Bonola C, Camagni P, Chiodelli P, Samoggia G (1991) Radiation Effects and Defects in Solids 119121:457-462.

12. Rutman J, Raz S, Riess I (2006) Solid State lonics 177:1771-1777.

13. Janek J, Korte C (1999) Solid State Ionics 116:181-195.

14. Farley JM, Thorp JS, Ross JS, Saunders GA (1972) J Mat Sci 7:475-476.

15. Luerssen B, Janek J, Günter S, Kiskinova M, Imbihl R (2002) Phys Chem Chem Phys 4:2673-2679.

16. Siegel DA, El Gabaly F, McCarty KF, Bartelt NC (2015) Phys Rev B 92:125421.

17. Kreka K, Hansen KV, Mogensen MB, Norrman K, Chatzichristodoulou C, Jacobsen T (2018) J Electrochem Soc 165:F253-F263.

18. Stalick JK, Waterstrat RM (2007) J Alloys and Compounds 430:123-131.

19. Wu Y, Hansen KV, Jacobsen T, Mogensen M (2011) Solid State lonics 197:32-36.

20. Hansen KV, Sander C, Koch S, Mogensen M (2007) J Phys Conf Ser 61:389-393.

21. Hansen KV, Wu Y, Jacobsen T, Mogensen MB, Theil Kuhn L (2013) Rev Sci Instrum 84:073701-0737017.

22. Huber TM, Opitz A, Kubicek M, Hutter H, Fleig J (2014) Solid State lonics 268:82-93.

23. Hansen KV, Norrman K, Jacobsen T (2016) Ultramicroscopy 170:69-76.

24. Robie RA, Hemingway BS (1995) U S Geological Survey Bulletin 2131.

25. Boukamp BA (2001) Solid State lonics 143:47-55.

26. Adler SB (2002) J Electrochem Soc 149:E166-E172.

27. Masó N, West A (2015) Chem Mater 27:1552-1558.

28. Park J-H, Blumenthal RN (1989) J Electrochem Soc 136:2867-2876.

29. Appel CC, Bonanos N, Horsewell A, Linderoth S (2001) J Mater Sci 36:4493-4501.

30. Holm R (1967) Stationary Contacts. In: Electric contacts (4. ed), Springer-Verlag, Berlin,pp 18-

31. Newman J (1966) J Electrochem Soc 113:501-503.

32. Schefold J, Brisse A, Zahid M (2009) J Electrochem Soc 156:B897-B904.

33. Klotz D, Szász J, Weber A, Ivers-Tiffée E (2012) ECS Trans 45:241-249.

34. Jacobsen T, Mogensen M (2008) ECS Trans 13:259-273.

35. Jamnik J, Maier J (2001) Phys Chem Chem Phys 3:1678.

36. Cole KS (1965) Physiol Rev 45:340-379.

37. Sandblom J, Walker JL, Eisenman G (1972) Biophys J 12:587-596. 
38. Sistat P, Kozmai A, Pismenskaya N, Larchet C, Popurcelly G, Nikonenko V (2008) Electrochim Acta 53:6380-6390.

39. Taibl S, Fafilek G, Fleig J (2016) Nanoscale 8:13954-13966.

40. Schouler EJL, Kleitz M (1987) J Electrochem Soc 134:1045-1050.

41. Tao Y, Shao J, Cheng S (2016) ACS Appl Mater Interfaces 8:17023-17027.

42. Hansen KV, Norrman K, Mogensen M (2006) Surf Interface Anal 38:911-916. 